\title{
Cultural Dance Program Improves Hypertension Management for Native Hawaiians and Pacific Islanders: a Pilot Randomized Trial
}

\author{
Joseph Keawe'aimoku Kaholokula ${ }^{1} \cdot$ Mele Look $^{1,2} \cdot$ Tricia Mabellos $^{1}$ • \\ Guangxiang Zhang ${ }^{1}$ - Mapuana de Silva ${ }^{2}$ - Sheryl Yoshimura ${ }^{3}$ - Cappy Solatorio ${ }^{4}$. \\ Thomas Wills ${ }^{5}$. Todd B. Seto ${ }^{6}$ - Ka ${ }^{6}$ imi A. Sinclair ${ }^{7}$
}

Received: 19 May 2015 / Revised: 29 July 2015 / Accepted: 27 November 2015 / Published online: 22 December 2015

(C) The Author(s) 2015. This article is published with open access at Springerlink.com

\begin{abstract}
Objective Native Hawaiians and Pacific Islanders (NHPI) bear an unequal burden of hypertension and cardiovascular disease. Hula, the traditional dance of Hawaii, has shown to be a culturally meaningful form of moderate-vigorous physical activity for NHPI. A pilot study was done in Honolulu, Hawaii, to test a 12-week hula-based intervention, coupled with self-care education, on blood pressure management in NHPI with hypertension in 2013.

Method NHPI with a systolic blood pressure (SBP) $\geq 140 \mathrm{mmHg}$ were randomized to the intervention $(n=27)$ or a wait-list control $(n=28)$. Blood pressure, physical functioning, and eight aspects of health-related quality of life (HRQL) were assessed.

Results The intervention resulted in a reduction in SBP compared to control ( -18.3 vs. $-7.6 \mathrm{mmHg}$, respectively, $p \leq 0.05$ ) from baseline to 3 -month post-intervention. Improvements in
\end{abstract}

Joseph Keawe'aimoku Kaholokula

kaholoku@hawaii.edu

1 Department of Native Hawaiian Health, University of Hawaii, John A. Burns School of Medicine, 677 Ala Moana Blvd, Ste 1016B, Honolulu, HI 96813, USA

2 Halau Mohala 'Ilima, 1110 A'alapapa Drive, Kailua, HI 96734, USA

3 Kokua Kalihi Valley Comprehensive Family Center, 2239 N. School St., Honolulu, HI 96819, USA

4 Kula No Na Po'e Hawai'i, 2150 Tantalus Drive, Honolulu, HI 96813, USA

5 Cancer Prevention and Control Program, University of Hawaii Cancer Center, 701 Ilalo Street, Honolulu, HI 96813, USA

6 The Queen's Medical Center, 1301 Punchbowl Street, Honolulu, HI 96813, USA

7 Department of Epidemiology, University of Washington, 1100 Olive Way, Ste 1200, Seattle, WA 98101, USA
HRQL measures of bodily pain and social functioning were significantly associated with SBP improvements in both groups. Conclusion Using hula as the physical activity component of a hypertension intervention can serve as a culturally congruent strategy to blood pressure management in NHPI with hypertension.

Trial registration clinicaltrials.gov Identifier: NCT01995812

Keywords Native Hawaiian and Pacific Islanders · Hypertension · Hula · Community-based participatory research $\cdot$ Blood pressure

\section{Introduction}

Hypertension, or chronic high blood pressure, is a serious public health concern in the USA, affecting $25 \%$ of the adult population [1]. It is a major risk factor for coronary heart disease (CHD) and the single most important risk factor for stroke, accounting for nearly $50 \%$ of ischemic strokes [2]. Native Hawaiians and other Pacific Islanders (NHPI) bear an unequal burden of hypertension and its consequences. Compared to Whites, they are $70 \%$ more likely to have hypertension, three and four times more likely to have CHD and stroke, respectively, and develop these diseases a decade sooner on average [3-5]. NHPI are also less likely to receive adequate hypertension treatment than Whites, leading to a greater risk of CHD and stroke [6, 7].

Western medical and behavioral approaches to hypertension management may not be as attractive to many NHPI than those that also include culturally relevant strategies, such as exercise programs based on their own cultural norms, and are sensitive to their cultural values and preferred modes of living [8-11]. Previous studies of NHPI found that they prefer 
traditional Pacific approaches to healing and treatment modalities that account for their unique spiritual and cultural values and delivered within a familiar community setting [11-14]. Many NHPI also have a distrust toward Western medicine that make them reluctant to participate in medical-focused health promotion programs [13]. Thus, hypertension interventions based on culturally meaningful health promotion strategies could lead to more NHPI participating in such interventions and lead to sustainable behavior change because of greater relevance to their lived cultural context. Given the higher risk of CHD and stroke in NHPI than other ethnic groups, and at younger ages, it is imperative that alternative and innovative strategies to hypertension management be developed and tested.

Physical activity is a major part of behavioral lifestyle interventions to both prevent and treat hypertension [15, 16]. Increased physical activity is associated with reduction in systolic blood pressure (SBP) of 5 to $10 \mathrm{mmHg}$ and in diastolic blood pressure (DBP) of 1 to $6 \mathrm{mmHg}$ in hypertensive patients [17], which is comparable to the effects of sodium restriction [18] and weight reduction [19]. A reduction of $5.5 \mathrm{mmHg}$ in SBP and $3.0 \mathrm{mmHg}$ in DPB has been found to lower the risk of CHD by $15 \%$, stroke by $27 \%$, and all-cause mortality by $7 \%$ [20, 21]. The effects of physical activity can be magnified when coupled with other lifestyle changes, such as sodium reduction and weight loss, and are likely synergistic with pharmacologic interventions [22].

Despite the benefits of physical activity, many NHPI find it difficult to engage in regular physical activity because of socioeconomic barriers [23] and because many physical activity programs are individually based and less appealing, e.g., walking on a treadmill $[24,25]$. Previous studies find that NHPI desire culturally relevant physical activity programs that are consistent with their ethno-cultural values, such as group-based activities that foster a mind-body connection $[11,12]$. Tai chi, a mind-body physical activity originating out of Chinese martial arts, has been found to reduce SBP by 9.3 to $14.3 \mathrm{mmHg}$ and DPB by 6.0 to $7.2 \mathrm{mmHg}$ among hypertensive patients [26].

Hula is the traditional dance of Native Hawaiians, the indigenous people of Hawaii, and a hallmark of Hawaiian culture today. It is performed by men and women of all ages. Much like tai chi, hula appeals to a wide range of people from different ethnic groups and has spread beyond Hawaii to places such as Japan, Mexico, and Europe [27]. Originally performed to convey history, spiritual beliefs, and one's connection to the natural world, hula is now practiced as a form of cultural and creative expression. The dances of hula are comprised of specific controlled rhythmic movements that illustrate the meaning or poetry of the accompanying songs or chants $[27,28]$. They can vary in intensity and duration depending on the choreography of the dance, tempo of the music, and skill level of the dancer and can be modified to accommodate people who have physical limitations.

Hula has been found to be suitable as a form of physical activity with metabolic equivalents (METs) of 5.7 (range 3.17-9.77) and 7.55 (range 4.43-12.0) for moderateintensity and high-intensity physical activity, respectively [29]. METs ranging from 3.0 to 6.0 (i.e., expends 3.5 to $7 \mathrm{kcal} / \mathrm{min}$ ) are indicative of moderate physical activity while greater than 6.0 (i.e., expends $>7 \mathrm{kcal} / \mathrm{min}$ ) are indicative of vigorous physical activity [30]. As suggested of tai chi [31, 32], hula may also have stress-reducing benefits because it includes music and rhythmic movements and often performed in groups, which could provide social support [11]. Some studies have found that social support [33, 34] and music therapy [35] improves hypertension management. Thus, hula combines several aspects - moderate physical activity, social support opportunities, and other stress-reducing qualitiesimportant to hypertension management.

To improve hypertension management in NHPI, the academic and community researchers of the Hula Empowering Lifestyle Adaptations (HELA) Project designed a hypertension program with hula as its physical activity component, called Ola Hou i ka Hula (Ola Hou) translated as "renewed life through hula" $[8,10]$. We conducted a pilot randomized controlled trial (RCT) to test both feasibility and efficacy of Ola Hou in reducing the blood pressure in a community sample of NHPI with physician-diagnosed hypertension. We hypothesized that Ola Hou would lead to greater reductions in blood pressure when compared to a wait-list intervention control group. We further hypothesized that Ola Hou would improve both physical and social functioning and that these improvements would be associated with blood pressure reductions.

\section{Method}

\section{Study Design}

We conducted a pilot study using a 2-arm RCT with a wait-list control group to test the effects of our 12-week Ola Hou intervention on blood pressure in NHPI with physiciandiagnosed hypertension. This pilot study was conducted between October 2012 and June 2013. Figure 1 presents the CONSORT diagram and depicts the overall study design. We used a community-based participatory research (CBPR) approach in designing and implementing this study [8]. Our community investigators included a kumu hula (hula expert) and two NHPI community leaders, while our academic investigators included a cardiologist, psychologist, and public health researchers. Because this was a pilot study to also determine if the intervention was feasible for the community partners to implement in their respective community settings 


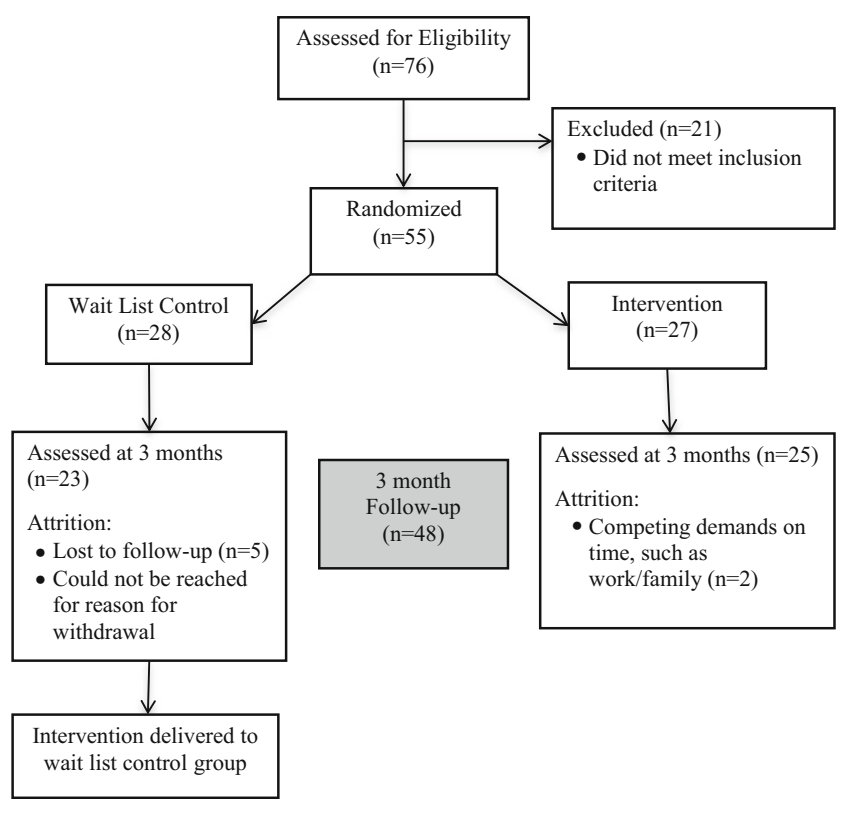

Fig. 1 CONSORT diagram of Ola Hou i ka Hula study participation

using their own community resources (e.g., kumu hula), we limited the data collection and measures to those essential to establish efficacy. Thus, we relied on randomization to balance participant unmeasured characteristics that might influence the outcomes, such as difference in hypertension medications prescribed and used.

\section{Participants}

Participants with diagnosed hypertension were recruited from two community-based organizations: a community health center primarily serving immigrant Pacific Islanders and a Native Hawaiian residential community center. The enrollment goal was 60 NHPI with 1:1 randomization within each community site. Eligibility criteria were (1) under a physician's care for $\geq 6$ months for hypertension, (2) continued to have indications of hypertension (SBP $>140$ or $>130$ if have co-morbid diabetes), (3) $\geq 21$ years of age, and (4) independently ambulatory. Exclusion criteria were (1) being prescribed more than four hypertension medications, (2) severe cognitive dysfunction precluding informed consent and understanding the intervention protocols, and/or (3) pregnancy at time or during the study period.

\section{Primary Outcome Measures}

The primary outcome or endpoint of this study was blood pressure $(\mathrm{mmHg})$, which was measured with an automatic blood pressure machine (Omron@HEM-907XL, Omron Healthcare, Palatine, Illinois). We used standardized protocols for obtaining blood pressure [36, 37]. For each assessment, SBP and DBP were measured in the sitting position and the participant was asked to sit quietly for $2-5$ min before measuring blood pressure. Both SBP and DBP were measured three times from each participant at each assessment occasion, and the average of the three at each was used for data analyses. Only SBP was considered as the primary outcome for this study since participants' eligibility was based only on their SBP being in the hypertensive range. However, DBP was also collected and analyzed.

\section{Secondary Outcome Measures}

A secondary outcome or endpoint was physical functioning as measured by the 6 -min walk test (6MWT), which measures the distance a person is able to walk in $6 \mathrm{~min}$ [38]. Participants were asked to perform the 6MWT using a fixed lap distance of either 60 or $100 \mathrm{ft}$. Each participant was instructed to walk as briskly as possible (without running) for $6 \mathrm{~min}$. Instructions and prompts for the 6MWT are based on standardized protocols. It has been found to be a valid test to estimate physical functioning [38] and has been used in other RCT with NHPI [39].

Another secondary outcome or endpoint was health-related quality of life (HRQL) as measured by the Medical Outcomes Study 12-Item Short Form Health Survey (SF-12) [40]. The SF-12 has eight subscales that assess facets of a person's HRQL: (1) physical functioning, (2) role physical functioning, (3) bodily pain, (4) general health, (5) vitality, (6) social functioning, (7) role-emotional functioning, and (8) mental health. Each subscale score, after transformation, ranges from 0 to 100 , with a higher score indicating a better health status. The SF-12 has been extensively used and found to be a reliable and valid measure of HRQOL across different populations [41].

\section{Demographic and Body Mass Index}

Demographic variables and body mass index (BMI) were assessed for baseline comparison to ensure balanced randomization and for possible statistical adjustments in case of an imbalance. Demographic variables were collected using a brief questionnaire asking for respondent's age, birthdate, sex (male or female), ethnicity (Native Hawaiian, other Pacific Islander, or other non-Pacific Islander ethnic groups), past experience dancing hula (yes or no), history of heart disease (e.g., heart attack or heart failure), history of other medical conditions (e.g., diabetes), and if they were using any prescribed hypertension medication (yes or no). Weight in kilograms and height in centimeters were measured of each participant to calculate BMI (i.e., $\mathrm{kg} / \mathrm{m}^{2}$ ).

\section{Procedures and Intervention Description}

The University of Hawaii Committee on Human Studies approved the study protocol. Community health workers 
recruited potential participants through flyers posted in their respective community settings, community health fairs, and by word of mouth. After individuals were screened, enrolled, and completed their baseline assessment, they were randomized 1:1 within each of the two community-based organizations to either the Ola Hou intervention or to the wait-list intervention control group. Participants in both study arms were instructed to continue receiving usual care from their physician and that their participation in this study did not replace the need for routine medical care. All participants obtained their physicians' approval for their participation in moderate physical activity.

Participants randomly assigned to the wait-list group did not receive the Ola Hou program and its educational materials, and no contact was made with them during the time they were on the wait-list. However, they were not restricted from seeking out other interventions, educational materials, or help on their own. They were free to perform, on their own, any type of exercise during this time and participate in any other health promotion program.

Participants assigned to the Ola Hou program received $3 \mathrm{~h}$ of hypertension education and 12 weeks of hula instruction and training consisting of two 60-min classes per week: The 3-h Ola Hou hypertention education curriculum was previously developed and culturally adapted for NHPI based on the Heart Failure Society of America's educational guidelines and modules [42]. The curriculum included four modules: (1) signs and symptoms of hypertension, (2) managing medication, (3) heart healthy eating to include sodium reduction, and (4) physical activity and managing negative emotions. Community health workers led the education sessions and brief interactive activities, such as cooking demonstrations of healthy recipes of NHPI ethnic foods and practicing of self-monitoring and stress management strategies. This education curriculum was delivered in six, 30-min sessions immediately after the 60min hula sessions from week 2 through week 7 . Each hula class consisted of 12 to 15 participants, which provided them with the opportunity to develop and engage in a social support network. The specific dances used in this study were evaluated and found to be an appropriate level of physical activity based on national recommendations [43, 44] and for individuals with limited mobility and fitness [8]. Classes were delivered by a kumu hula and included the phases and goals described in Table 1.

The design of the Ola Hou program was informed by the social cognitive theory [45], which has been used successfully to inform many types of physical activity interventions [46]. It is primarily based on the principle of reciprocal determinism, referring to the bidirectional behaviorenvironment interaction on influencing behavior. It posits that a person's knowledge or behavior acquisition is directly related to the observation of others (or models) within his or her context of social interactions and experiences. It is through these social observations and interactions that a person's self-efficacy to make and sustain behavior change is achieved and reinforced.

All participants, both those in the intervention and wait-list control, were assessed by study staff using standardized protocols to obtain clinical and demographic and psychosocial information through in-person interviews. Assessments were performed at baseline and 3-months post-intervention. All participants received a $\$ 25$ gift card after the completion of each assessment period. Intervention participants received incentives such as cooking supplies, bags, and water bottles at various time points during the Ola Hou program.

\section{Statistical Analysis}

The baseline demographics, SBP, DBP, 6MWT, and SF-12 subscale scores were summarized by descriptive statistics: means $(\mathrm{M})$ and standard deviations $(\mathrm{SD})$ for continuous variables (i.e., age, BMI, SBP, DBP, 6MWT, and SF-12 subscale scores) and frequencies and proportions for categorical variables [i.e., sex (female vs. male), ethnic group (Native Hawaiian, other Pacific Islander, or other ethnic group), heart disease (no vs. yes), other medical conditions (no vs. yes), and prescribed hypertension medication (no vs. yes)]. Comparisons between the two study arms at baseline on the aforementioned variables were conducted using two sample $t$ test, chi-squared, or Fisher's exact tests, as appropriate. The retention rates between two groups were evaluated by Fisher's exact test.

Multivariable linear regression modeling were used to examine change in our primary endpoints of SBP and DBP (primary outcomes), separately, from baseline to 3-month assessment (i.e., 3-month BP-baseline BP; continuous change variable) between study arms while adjusting for age, baseline blood pressure, and baseline characteristics (i.e., presence of heart disease or not; see Table 2$)$ that were notably $(p<0.10)$ different between groups. Age was adjusted, as recommended for hypertension outcome studies $[1,15]$. It is also convention when examining the effects of a RCT intervention to adjust for the baseline value of the primary outcome when change from baseline is the primary endpoint. Both intention-to-treat and complete case analyses were done for SBP and DPB each using multivariable linear regression modeling.

Intention-to-treat analyses were performed for all participants randomized, with missing values at 3 -month assessments imputed by baseline observation values carried forward. Complete case analyses were also conducted, which included only the participants with both baseline and 3month assessment data available. Thus, seven participants with only baseline assessment data but without 3-month data were excluded in the complete case analyses.

To further evaluate the clinical impact of the Ola Hou program on hypertension control, participants in both study arms 
Table 1 Design of Ola Hou physical activity program

\begin{tabular}{|c|c|c|}
\hline Phase & Duration & Goals \\
\hline Warm-up & $5-15 \mathrm{~min}$ & $\begin{array}{l}\text { - Promote normal range of motion } \\
\text { - Stretches focusing on legs, arms, lower back } \\
\text { - Low-level, aerobic activity at } 25-40 \% \text { MPHR }\end{array}$ \\
\hline Conditioning & $20-40 \mathrm{~min}$ & $\begin{array}{l}\text { Intensity } \\
\text { - Range } 40-85 \% \mathrm{VO}_{\max } \text { or } 50-70 \% \mathrm{MPHR} \\
\text { - Training intensity }=(40+[2 \times \mathrm{Max} \mathrm{METs}]) \% \\
\cdot \text { RPE: between } 12 \text { and } 16 \text { on Borg scale } \\
\text { - Target } \mathrm{HR}=(\mathrm{HR} \text { reserve } \times \text { training intensity }[\%])+\mathrm{HR}_{\text {resting }}\end{array}$ \\
\hline Cool-down & $3-10 \mathrm{~min}$ & - Low-level, aerobic activity to allow BP and HR to return to resting level \\
\hline
\end{tabular}

MPHR maximum predicted heart rate, $V O_{\max }$ maximal oxygen consumption, $M E T s$ metabolic equivalents, $R P E$ rating of perceived exertion, $H R$ heart rate, $B P$ blood pressure

${ }^{a}$ The Borg scale is commonly used in cardiac rehabilitation as a subjective measure of physical exertion. The participant gives a subjective score between 6 and 20 during exercise to indicate his or her level of physical intensity [45]

were categorized based on those with a reduction of $\mathrm{SBP} \geq 10 \mathrm{mmHg}$ versus those without. A reduction of $\geq 10 \mathrm{mmHg}$ in SBP over 3 months was regarded as being clinically significant (i.e., reducing the risk for CHD and stroke) based on the findings of previous studies [20,21]. Chi-squared $\left(\chi^{2}\right)$ analysis was used to compare between participants of the two study arms based on this SBP categorization.

Multivariable linear models were also used to examine the change in our secondary endpoints, separately, from baseline to 3-month assessment (i.e., 3-month value-baseline value) between study arms while adjusting for age, baseline value of the relevant secondary endpoint, and baseline characteristics (i.e., presence of heart disease or not; see Table 2) that were notably $(p<0.10)$ different between groups. Both intention-totreat and complete case analyses were also done for these analyses.

We also examined the association between the changes in secondary endpoints and the changes in SBP (the primary endpoint) from baseline to 3-month assessment in the entire sample combined. These associations were evaluated by Pearson correlation coefficients. These analyses were done to determine what physical functioning and HRQL variables significantly co-varied with SBP regardless of study arm.

Formal sample size calculations were not performed for this pilot study. However, sample size was estimated based on a review of literature of similar mind and body physical activities used in hypertension interventions [31, 32, 47, 48]. Study data were collected and managed using REDCap electronic data capture tools [49] hosted at the University of Hawaii John A. Burns School of Medicine. All statistical analyses were performed using SAS software version 9.3 (SAS Institute Inc., Cary, NC). A two-sided $p$ value $\leq 0.05$ was considered statistically significant.

\section{Results}

\section{Participants}

Table 2 summarizes the baseline characteristics, which were comparable for the most part between the participants of the two study arms. For both study arms, the mean age was 55 years $(\mathrm{SD}=10$ for Ola Hou and 12 for wait-list control participants) and a majority were NHPI (95\%) and female $(85 \%)$. More participants in the Ola Hou program $(41 \%)$ compared to control $(25 \%)$ had previously danced hula and had no previous history of heart disease (56 and $29 \%$, respectively), but these differences were not statistically significant. However, the difference between arms on the number of participants with "no heart disease" did approach significance $(p=0.06)$. Both study arms were balanced in regards to having "other medical conditions," to being on "prescribed hypertension medications," and to BMI, SBP, DBP, 6MWT, and scores on the SF-12 subscales.

\section{Retention}

Of the 55 participants assigned to the two study arms, 48 $(87 \%)$ completed the 3-month post-intervention assessment. There was no significant difference $(p=0.42)$ in attrition between the intervention arm $(n=2,7 \%)$ and the wait-list control $\operatorname{arm}(n=5,18 \%)$ at 3-month assessment.

\section{Primary Outcome Analyses}

Table 3 summarizes the results of the blood pressure outcomes by study arms. Ola Hou participants, compared to wait-list control participants, had greater reductions in their SBP in both the intention-to-treat analysis $(-18.3$ vs. 
Table 2 Baseline characteristics of participants by study group

\begin{tabular}{|c|c|c|c|}
\hline Variable & $\begin{array}{l}\text { Ola Hou } \\
(n=27)\end{array}$ & $\begin{array}{l}\text { Control } \\
(n=28)\end{array}$ & $\begin{array}{l}\text { Group differences } \\
(p \text { value })^{\mathrm{a}}\end{array}$ \\
\hline Age in years, $M(S D)$ & $55(10)$ & $55(12)$ & 0.90 \\
\hline Female, $n(\%)$ & $25(93)$ & $22(79)$ & 0.26 \\
\hline Ethnic group, $n(\%)$ & & & 1.00 \\
\hline Native Hawaiian & $13(48)$ & $14(50)$ & \\
\hline Other Pacific Islander & $13(48)$ & $12(43)$ & \\
\hline Other ethnic group & $1(4)$ & $2(7)$ & \\
\hline Danced hula, $n(\%)$ & $11(41)$ & $7(25)$ & 0.26 \\
\hline \multicolumn{4}{|l|}{ Heart disease, $n(\%)$} \\
\hline Heart attack & $0(0)$ & $2(7)$ & 0.50 \\
\hline Heart failure & $0(0)$ & $1(4)$ & 1.00 \\
\hline High cholesterol & $12(44)$ & $19(68)$ & 0.11 \\
\hline No heart disease & $15(56)$ & $8(29)$ & 0.06 \\
\hline \multicolumn{4}{|l|}{ Other medical conditions, $n(\%)$} \\
\hline Diabetes & $16(59)$ & $15(54)$ & 0.79 \\
\hline Other medical conditions & $7(26)$ & $7(25)$ & 1.00 \\
\hline No other medical conditions & $6(22)$ & $7(25)$ & 1.00 \\
\hline Prescribed hypertension medications, $n(\%)$ & $20(74)$ & $25(89)$ & 0.18 \\
\hline $\mathrm{BMI}, \mathrm{kg} / \mathrm{m}^{2}, M(\mathrm{SD})$ & $36(7)$ & $39(6)$ & 0.11 \\
\hline SBP, mmHg, $M$ (SD) & $148(14)$ & $143(12)$ & 0.16 \\
\hline $\mathrm{DBP}, \mathrm{mmHg}, M(\mathrm{SD})$ & $85(12)$ & $83(13)$ & 0.71 \\
\hline 6MWT, ft, $M(\mathrm{SD})$ & $1264(191)$ & $1336(261)$ & 0.26 \\
\hline \multicolumn{4}{|l|}{$\mathrm{SF}-12$ subscales, $M(\mathrm{SD})^{\mathrm{b}}$} \\
\hline Physical functioning & $46(40)$ & $50(42)$ & 0.74 \\
\hline Role physical functioning & $48(28)$ & $41(33)$ & 0.43 \\
\hline Bodily pain & $63(28)$ & $71(17)$ & 0.23 \\
\hline General health & $48(20)$ & $56(20)$ & 0.15 \\
\hline Vitality & $57(25)$ & $63(28)$ & 0.48 \\
\hline Social functioning & $52(32)$ & $66(31)$ & 0.10 \\
\hline Role-emotional functioning & $51(34)$ & $56(38)$ & 0.62 \\
\hline Mental health & $58(20)$ & $63(17)$ & 0.28 \\
\hline
\end{tabular}

$M$ mean, $S D$ standard deviation, $B M I$ body mass index, $S B P$ systolic blood pressure, $D B P$ diastolic blood pressure

${ }^{a}$ Group difference $p$ values based on two sample independent $t$ tests and chi-squared or Fisher's exact tests, as appropriate

${ }^{\mathrm{b}}$ SF-12 subscale scores are calculated as a percentage ( 0 to $100 \%$ ) with higher scores indicating better health and well-being
$-7.6 \mathrm{mmHg}$, respectively) and the complete case analysis ( -19.8 vs. $-9.2 \mathrm{mmHg}$, respectively) from baseline to 3month assessment. After adjusting for age, heart disease status, and baseline blood pressure, there were significant differences at 3 months between the Ola Hou and the waitlist control group for SBP, in both the intention-to-treat $[F(1,50)=4.19, p=0.046]$ and complete case analyses for $\operatorname{SBP}[F(1,43)=4.24, p=0.045]$. There were no significant differences in DBP between the intervention and control participants from baseline to 3-month assessment. The invention group also had significantly more participants with at least $10 \mathrm{mmHg}$ reduction in SBP at 3-month assessment than those in the wait-list control group [72 vs. $39 \%$, respectively; $\left.\chi^{2}(1, n=48)=5.26, p=0.022\right]$.

\section{Secondary Outcome Analyses}

Table 4 summarizes the results of the physical functioning and self-reported HRQL outcomes by study arms. Adjusting for age, heart disease status, and baseline value of the secondary outcome variable, there were no significant differences between the study arms on the 6MWT and SF-12 subscales in change from baseline to 3-month assessment based on either intention-to-treat analysis or complete case analysis. 
Table 3 Intervention effects on blood pressure from baseline to 3 month follow-up ${ }^{\mathrm{a}}$

\begin{tabular}{|c|c|c|c|c|c|}
\hline & $\begin{array}{l}\text { Baseline } \\
M(S D)\end{array}$ & $\begin{array}{l}3 \text { month } \\
M(S D)\end{array}$ & Change $\pm S E$ & $F$ test & $\begin{array}{l}\text { Group differences } \\
(p \text { value })^{\mathrm{b}}\end{array}$ \\
\hline \multicolumn{6}{|l|}{ Systolic blood pressure } \\
\hline Intention-to-treat $^{\mathrm{c}}$ & & & & $F(1,50)=4.19$ & 0.046 \\
\hline Ola Hou $(n=27)$ & $148.4(14.2)$ & $130.1(17.3)$ & $-18.3 \pm 3.2$ & & \\
\hline Control $(n=28)$ & $143.4(11.8)$ & $135.8(18.0)$ & $-7.6 \pm 3.0$ & & \\
\hline Complete cases ${ }^{\mathrm{d}}$ & & & & $F(1,43)=4.24$ & 0.045 \\
\hline Ola Hou $(n=25)$ & $147.9(13.4)$ & $128.1(15.1)$ & $-19.8 \pm 3.3$ & & \\
\hline Control $(n=23)$ & $144.1(10.4)$ & $134.9(18.2)$ & $-9.2 \pm 3.6$ & & \\
\hline \multicolumn{6}{|c|}{ Diastolic blood pressure } \\
\hline Intention-to-treat $^{\mathrm{c}}$ & & & & $F(1,50)=0.80$ & 0.38 \\
\hline Ola Hou $(n=27)$ & $84.6(12.1)$ & $79.0(10.9)$ & $-5.6 \pm 1.6$ & & \\
\hline Control $(n=28)$ & $83.4(12.6)$ & $80.5(13.1)$ & $-2.9 \pm 2.0$ & & \\
\hline Complete cases ${ }^{\mathrm{d}}$ & & & & $F(1,43)=0.98$ & 0.33 \\
\hline Ola Hou $(n=25)$ & $84.2(12.1)$ & $78.1(10.4)$ & $-6.1 \pm 1.7$ & & \\
\hline Control $(n=23)$ & $83.7(11.2)$ & $80.2(11.9)$ & $-3.5 \pm 2.4$ & & \\
\hline
\end{tabular}

$M$ mean, $S D$ standard deviation, $S E$ standard error

${ }^{a}$ Unadjusted descriptive means (SD) and changes (SE) are reported in the table

${ }^{\mathrm{b}}$ Based on multivariable linear model with the blood pressure change (3-month value minus baseline value) as the dependent variable, adjusting for the baseline blood pressure value, age, and heart disease status (presence vs. absence)

${ }^{\mathrm{c}}$ Intention-to-treat analyses with missing value of blood pressure at 3-month assessment imputed by individual participant's baseline observation value carried forward

${ }^{\mathrm{d}}$ Complete case analyses included only participants with both baseline and 3-month assessment data available $(n=48)$
However, it is important to note that greater average improvements on these variables were found in the Ola Hou participants compared to the wait-list control participants, and the difference between them on the bodily pain and social functioning scores did approach significance $(p<0.08)$. It is also important to note that on five of the eight, the scores on the SF12 subscales (i.e., bodily pain, general health, vitality, social functioning, and mental health) worsened, on average, from baseline to 3-month assessment for the wait-list control group.

\section{Association between SBP and Secondary Outcomes}

For both Ola Hou and wait-list control participants, improvements in bodily pain $[r(46)=-0.32, p=0.03]$ and social functioning scores [ $r(46)=-0.50, p=0.0003]$ were significantly associated with improved change in SBP from baseline to 3month assessment (data not shown in tables). None of the other SF-12 subscale scores were significantly associated with the improved change in SBP from baseline to 3-month assessment.

\section{Discussion}

We examined the efficacy of a hula-based intervention in improving blood pressure control, physical functioning, and HRQL in a community-based sample of NHPI with a confirmed diagnosis of hypertension. In partial support of our hypothesis, we found that the hula-based Ola Hou program led to significantly greater reductions in SBP and notable improvements in bodily pain when compared to those randomized to the wait-list intervention control group. Improvements in both bodily pain and social functioning were significantly associated with improvements in SBP in both groups. However, there were no statistically significant differences between the intervention and control group on measures of physical functioning and HRQL.

We found that the Ola Hou Program led to a greater reduction in SBP $(-9.8 \mathrm{mmHg})$ over the 3-month intervention period. This improvement is comparable to other studies of physical activity-based interventions [17] and the use of tai chi [26] for hypertension management. The hula-based intervention led to a reduction in SBP of $10 \mathrm{mmHg}$ or greater for $72 \%$ of the intervention participants compared to $39 \%$ of those in wait-list control. A $5 \mathrm{mmHg}$ reduction in SBP has been found to lower a person's risk of ischemic heart disease by $21 \%$, stroke by $34 \%$, and all-cause mortality by $7 \%$ [20, 50]. This magnitude of reduction in SBP exceeds the reduction typically seen with antihypertensive medication monotherapy [51]. We did not find significant improvements in DBP in this study, which is likely due to the fact that we based 
Table 4 Intervention effects on secondary outcomes at baseline and 3-month follow-up ${ }^{\mathrm{a}}$

\begin{tabular}{|c|c|c|c|c|c|}
\hline & $\begin{array}{l}\text { Baseline, } \\
M(S D)\end{array}$ & $\begin{array}{l}3 \text { month, } \\
M(S D)\end{array}$ & $\begin{array}{l}\text { Change } \\
\pm S E\end{array}$ & $F$ test & $\begin{array}{l}\text { Group differences } \\
(p \text { value })^{\mathrm{b}}\end{array}$ \\
\hline \multicolumn{6}{|l|}{ 6-Min walk test, $\mathrm{ft}$} \\
\hline Intention-to-treat ${ }^{\mathrm{c}}$ & & & & $F(1,50)=0.003$ & 0.96 \\
\hline Ola Hou $(n=27)$ & $1264.4(190.6)$ & $1308.9(278.7)$ & $44.4 \pm 37.3$ & & \\
\hline Control $(n=28)$ & $1335.8(261.0)$ & $1351.1(260.1)$ & $15.3 \pm 38.1$ & & \\
\hline Complete cases ${ }^{\mathrm{d}}$ & & & & $F(1,43)=0.04$ & 0.84 \\
\hline Ola Hou $(n=25)$ & $1263.6(198.3)$ & $1311.6(289.8)$ & $48.0 \pm 40.3$ & & \\
\hline Control $(n=23)$ & $1384.9(252.1)$ & $1403.5(247.0)$ & $18.6 \pm 46.5$ & & \\
\hline \multicolumn{6}{|l|}{ Physical functioning } \\
\hline Intention-to-treat ${ }^{\mathrm{c}}$ & & & & $F(1,50)=0.12$ & 0.73 \\
\hline Ola Hou $(n=27)$ & $46.3(39.7)$ & $53.7(38.4)$ & $7.4 \pm 5.9$ & & \\
\hline Control $(n=28)$ & $50.0(41.9)$ & $50.0(36.0)$ & $0.0 \pm 6.2$ & & \\
\hline Complete cases ${ }^{\mathrm{d}}$ & & & & $F(1,43)=0.04$ & 0.84 \\
\hline Ola Hou $(n=25)$ & $50.0(38.9)$ & $58.0(36.6)$ & $8.0 \pm 6.4$ & & \\
\hline Control $(n=23)$ & $56.5(42.1)$ & $56.5(34.7)$ & $0.0 \pm 7.5$ & & \\
\hline \multicolumn{6}{|l|}{ Role physical } \\
\hline Intention-to-treat $^{c}$ & & & & $F(1,50)=0.38$ & 0.54 \\
\hline Ola Hou $(n=27)$ & $47.7(28.0)$ & $60.3(24.9)$ & $12.5 \pm 5.1$ & & \\
\hline Control $(n=28)$ & $41.1(33.3)$ & $52.2(29.9)$ & $11.2 \pm 4.0$ & & \\
\hline Complete cases $^{\mathrm{d}}$ & & & & $F(1,43)=0.10$ & 0.75 \\
\hline Ola Hou $(n=25)$ & $50.5(26.9)$ & $64.0(26.3)$ & $13.5 \pm 5.4$ & & \\
\hline Control $(n=23)$ & $46.7(32.9)$ & $60.3(24.9)$ & $13.6 \pm 4.7$ & & \\
\hline \multicolumn{6}{|l|}{ Bodily pain } \\
\hline Intention-to-treat ${ }^{\mathrm{c}}$ & & & & $F(1,50)=3.19$ & 0.08 \\
\hline Ola Hou $(n=27)$ & $63.0(28.1)$ & $72.2(19.5)$ & $9.3 \pm 4.7$ & & \\
\hline Control $(n=28)$ & $70.5(16.7)$ & $66.1(22.8)$ & $-4.5 \pm 4.1$ & & \\
\hline Complete cases ${ }^{\mathrm{d}}$ & & & & $F(1,43)=3.62$ & 0.064 \\
\hline Ola Hou $(n=25)$ & $62.0(29.0)$ & $72.0(19.5)$ & $10.0 \pm 5.0$ & & \\
\hline Control $(n=23)$ & $70.7(17.9)$ & $65.2(24.7)$ & $-5.4 \pm 5.0$ & & \\
\hline \multicolumn{6}{|l|}{ General health } \\
\hline Intention-to-treat ${ }^{c}$ & & & & $F(1,50)=0.75$ & 0.39 \\
\hline Ola Hou $(n=27)$ & $47.8(20.1)$ & $54.6(18.4)$ & $6.9 \pm 3.6$ & & \\
\hline Control $(n=28)$ & $55.7(20.4)$ & $53.8(17.2)$ & $-2.0 \pm 3.2$ & & \\
\hline Complete cases ${ }^{\mathrm{d}}$ & & & & $F(1,43)=0.74$ & 0.40 \\
\hline Ola Hou $(n=25)$ & $46.8(20.6)$ & $54.2(19.1)$ & $7.4 \pm 3.8$ & & \\
\hline Control $(n=23)$ & $54.8(22.4)$ & $52.4(18.8)$ & $-2.4 \pm 3.9$ & & \\
\hline \multicolumn{6}{|l|}{ Vitality } \\
\hline Intention-to-treat ${ }^{\mathrm{c}}$ & & & & $F(1,50)=1.29$ & 0.26 \\
\hline Ola Hou $(n=27)$ & $57.4(24.8)$ & $60.2(18.7)$ & $2.8 \pm 5.4$ & & \\
\hline Control $(n=28)$ & $62.5(27.6)$ & $56.3(23.2)$ & $-6.3 \pm 4.7$ & & \\
\hline Complete cases ${ }^{\mathrm{d}}$ & & & & $F(1,43)=1.65$ & 0.21 \\
\hline Ola Hou $(n=25)$ & $55.0(23.9)$ & $58.0(17.3)$ & $3.0 \pm 5.8$ & & \\
\hline Control $(n=23)$ & $59.8(27.9)$ & $52.2(21.2)$ & $-7.6 \pm 5.8$ & & \\
\hline \multicolumn{6}{|l|}{ Social functioning } \\
\hline Intention-to-treat ${ }^{\mathrm{c}}$ & & & & $F(1,50)=3.29$ & 0.08 \\
\hline Ola Hou $(n=27)$ & $51.9(31.7)$ & $63.0(28.9)$ & $11.1 \pm 3.9$ & & \\
\hline Control $(n=28)$ & $66.1(31.3)$ & $60.7(30.8)$ & $-5.4 \pm 5.2$ & & \\
\hline Complete cases $^{\mathrm{d}}$ & & & & $F(1,43)=1.75$ & 0.19 \\
\hline Ola Hou $(n=25)$ & $55.0(30.6)$ & $67.0(25.7)$ & $12.0 \pm 4.1$ & & \\
\hline Control $(n=23)$ & $75.0(26.1)$ & $68.5(27.4)$ & $-6.5 \pm 6.3$ & & \\
\hline
\end{tabular}


Table 4 (continued)

\begin{tabular}{|c|c|c|c|c|c|}
\hline & $\begin{array}{l}\text { Baseline, } \\
M(S D)\end{array}$ & $\begin{array}{l}3 \text { month, } \\
M(S D)\end{array}$ & $\begin{array}{l}\text { Change } \\
\pm S E\end{array}$ & $F$ test & $\begin{array}{l}\text { Group differences } \\
(p \text { value })^{\mathrm{b}}\end{array}$ \\
\hline \multicolumn{6}{|l|}{ Role emotional } \\
\hline Intention-to-treat ${ }^{c}$ & & & & $F(1,50)=0.08$ & 0.80 \\
\hline Ola Hou $(n=27)$ & $51.4(34.0)$ & $58.3(29.0)$ & $6.9 \pm 6.0$ & & \\
\hline Control $(n=28)$ & $56.3(38.0)$ & $58.0(32.8)$ & $1.8 \pm 5.0$ & & \\
\hline Complete cases ${ }^{\mathrm{d}}$ & & & & $F(1,43)=0.06$ & 0.80 \\
\hline Ola Hou $(n=25)$ & $54.5(33.2)$ & $62.0(26.6)$ & $7.5 \pm 6.5$ & & \\
\hline Control $(n=23)$ & $65.2(34.7)$ & $67.4(26.8)$ & $2.2 \pm 6.1$ & & \\
\hline \multicolumn{6}{|l|}{ Mental health } \\
\hline Intention-to-treat ${ }^{\mathrm{c}}$ & & & & $F(1,50)=1.06$ & 0.31 \\
\hline Ola Hou $(n=27)$ & $57.8(20.0)$ & $63.4(17.7)$ & $5.6 \pm 3.6$ & & \\
\hline Control $(n=28)$ & $63.4(17.0)$ & $62.5(16.7)$ & $-0.9 \pm 2.2$ & & \\
\hline Complete cases $^{\mathrm{d}}$ & & & & $F(1,43)=0.91$ & 0.35 \\
\hline Ola Hou $(n=25)$ & $58.5(20.6)$ & 64.5 (17.9) & $6.0 \pm 3.9$ & & \\
\hline Control $(n=23)$ & $65.8(17.8)$ & $64.7(17.5)$ & $-1.1 \pm 2.7$ & & \\
\hline
\end{tabular}

$M$ mean, $S D$ standard deviation, $S E$ standard error

${ }^{a}$ Unadjusted descriptive means (SD) and changes (SE) are reported in the table

${ }^{\mathrm{b}}$ Based on multivariable linear model with the relevant variable change (3-month value minus baseline value) as the dependent variable, adjusting for the variable's baseline value, age, and heart disease status (presence vs. absence)

${ }^{\mathrm{c}}$ Intention-to-treat analyses with missing value at 3-month assessment imputed by individual participant's baseline observation value carried forward

${ }^{\mathrm{d}}$ Complete case analyses included only participants with both baseline and 3-month assessment data available $(n=48)$

eligibility for participation on systolic hypertension (>140 $\mathrm{mmHg}$ ); the participants, on average, had a DBP under $90 \mathrm{mmHg}$ at baseline.

The association between improved social functioning and decreased SBP for NHPI suggests another mechanism by which blood pressure can be affected, beyond the effects of physical activity itself. We found that social functioning was associated with SBP improvements for both groups. When comparing between groups, we found that social functioning, on average, improved for the intervention participants but slightly worsened over time for the control participants, although this difference was short of being statistically significant. However, it does provide support for the reciprocal determinism principle of the social cognitive theory [45]; that is, the social context can serve to influence a person's physical activity and other behavior changes [46]. We hypothesized that social functioning would improve as a result of participating in a hula-based intervention because hula is typically taught and practiced in a group setting and emphasizes synchronization in dancing. In addition, the hula training promotes the Hawaiian value of interconnectedness between individuals and fosters a family-like environment. It also emphasizes a mind-body approach by incorporating an understanding of the historical and cultural meaning behind the songs or chants that accompany the dance. The gestures and movements of hula are directly related to the words and their meaning, often metaphorically, which encourages coordination of mind and body to achieve a state of "being in the moment" and attuned to the symbolic meaning of one's movements.

As previously discussed, hula was found to achieve the METs expected of a moderate (5.7) to vigorous intensity (7.6) exercise and thus hypothesized to have benefits for hypertension management for NHPI [29]. The finding of our study supports this notion that hula, coupled with hypertension self-care education, can be effectively used for hypertension management among NHPI. Hula as a physical activity may also have synergistic effects with social support and social functioning and with the use of music and rhythmic motions that enhance hypertension control. Previous studies have found that social support [33, 34] and music [25] can lower blood pressure and may operate by stress reduction to lower sympathetic arousal $[52,53]$. Future studies are needed to elucidate the mechanism by which hula, as a mind-body physical activity approach, works to improve blood pressure in hypertension patients.

Notwithstanding the positive outcomes of our study, there are several methodological limitations worth noting. Aside from the 6MWT, we used subjective self-report measures of physical, emotional, and social functioning that are 
susceptible to response bias. However, it is unlikely that response bias was a problem in this study given that the more objective 6MWT along with most of the physical functioning self-report measures did not show significant improvements over the course of the intervention, although there was a trend toward improvement in most measures. We included NHPI with hypertension diagnosis based only on SBP. The effects of a hula-based intervention on DBP have yet to be determined. Another important limitation of our study is the absence of hypertension medication information to control for in the analysis. It is possible that those who participated in the Ola Hou program became more aware of their need to comply with their prescribed medication regimen. Although improved medication compliance is a desired outcome of the intervention, we were unable to determine if medication type or use may have influenced the differences we observed between study groups because of the pilot nature of our study. However, randomization should have balanced the two groups regarding medication use.

Overall, our study provides the strong preliminary evidence needed to more definitively test the effects of hula as a cultural dance to improve not only physical activity but other factors important to hypertension management in Native Hawaiians and Pacific Islanders. It also provides support for the investigation of other cultural forms of physical activity as the means to increase participation in health promotion interventions and the uptake and maintenance of regular physical activity that is culturally meaningful and sustainable in realworld settings. Finally, our study suggest multiple pathways to improving hypertension management in high-risk minority populations that is worthy of further investigation, such as comparing the relative effects of social functioning versus physical activity, in improving blood pressure control.

Funding The Ola Hou i ka Hula Project was supported by grants from the National Institute on Minority Health and Health Disparities (U54 MD007584, G12 MD007601, and P20 MD000173) and from National Institute of General Medical Sciences of the National Institutes of Health (P20 GM103466) and the Queen's Health Systems. The content is solely the responsibility of the authors and does not necessarily represent the official views of the National Institute on Minority Health and Health Disparities and National Institute of General Medical Sciences of the National Institutes of Health or the Queen's Health Systems.

\section{Compliance with Ethical Standards}

Conflict of Interest All authors declare that they have no conflict of interest.

Ethical Approval All procedures performed in studies involving human participants were in accordance with the ethical standards of the institutional and with the 1964 Helsinki Declaration and its later amendments or comparable ethical standards.

Informed Consent Informed consent was obtained from all individual participants included in the study.
Open Access This article is distributed under the terms of the Creative Commons Attribution 4.0 International License (http:// creativecommons.org/licenses/by/4.0/), which permits unrestricted use, distribution, and reproduction in any medium, provided you give appropriate credit to the original author(s) and the source, provide a link to the Creative Commons license, and indicate if changes were made.

\section{References}

1. American Heart Association. Understand your risk for high blood pressure. http://www.heart.org/HEARTORG/Conditions/ HighBloodPressure/UnderstandYourRiskforHighBloodPressure/ Understand-Your-Risk-for-High-Blood-Pressure_UCM_002052 Article.jsp Accessed January 6, 2014.

2. Seshadri S, Beiser A, Kelly-Hayes M, et al. The lifetime risk of stroke: estimates from the Framingham study. Stroke. 2006;37: 345-50. doi:10.1161/01.STR.0000199613.38911.b2.

3. Mau MK, Sinclair K, Saito EP, Baumhofer KN, Kaholokula JK. Cardiometabolic health disparities in native Hawaiians and other Pacific Islanders. Epidemiol Rev. 2009;31:113-29. doi:10.1093/ ajerev/mxp004.

4. Schiller JS, Lucas JW, Ward BW, Peregoy JA. Summary health statistics for U.S. adults: National Nealth Interview Survey, 2010. Vital Health Stat 10. 2012;252:1-207.

5. The Office of Minority Health $(\mathrm{OMH})$. Heart disease and Native Hawaiians/Pacific Islanders. http://minorityhealth.hhs.gov/ templates $/$ content.aspx $? 1 v l=3 \& 1 v 1 I D=6 \& I D=8654$. Accessed January 7, 2014

6. Aluli NE, Reyes PW, Brady SK, et al. All-cause and CVD mortality in Native Hawaiians. Diabetes Res Clin Pract. 2010;89(1):65-71. doi:10.1016/j.diabres.2010.03.003.

7. Nakagawa K, Koenig MA, Seto TB, Asai SM, Chang CW. Racial disparities among Native Hawaiians and Pacific Islanders with intracerebral hemorrhage. Neurology. 2012;79(7):675-80. doi:10. 1212/WNL.0b013e3182608c6f.

8. Look MA, Kaholokula JK, Carvahlo A, Seto T, de Silva M. Developing a culturally based cardiac rehabilitation program: the HELA study. Prog Community Health Partnersh. 2012;6(1):10310. doi:10.1353/cpr.2012.0012.

9. Roger VL, Go AS, Lloyd-Jones DM, et al. Executive summary: heart disease and stroke statistics-2012 update: a report from the American Heart Association. Circulation. 2012;125(1):188-97. doi:10.1161/CIR.0b013e3182456d46.

10. Look MA, Maskarinec GG, de Silva M, Seto T, Mau ML, Kaholokula JK. Kumu Hula perspectives on health. Hawai'i J Med Public Health. 2014;73(12 suppl 3):21-5.

11. Maskarinec GG, Look M, Tolentino K, Trask-Batti M, Seto T, de Silva M. Patient perspectives on the Hula Empowering Lifestyle Adaptations (HELA) study: benefits of dancing hula for cardiac rehabilitation. Health Promot Pract. 2015;16(1):109-14. doi:10. $1177 / 1524839914527451$.

12. Look MA, Batti-Trask MK, Agres R, Mau ML, Kaholokula JK. Assessment and priorities for health \& well-being in Native Hawaiians \& other Pacific peoples, Center for Native and Pacific Health Disparities Research. Honolulu HI: University of Hawai'i; 2013.

13. Kaholokula JK, Saito E, Mau M, Latimer R, Seto T. Pacific Islanders' perspectives on heart failure management. Patient Educ Couns. 2008;70(2):281-91.

14. Ka'opua LS, Park SH, Ward ME, Braun KL. Testing the feasibility of a culturally tailored breast cancer screening intervention with 
Native Hawaiian women in rural churches. Health Soc Work. 2011;36(1):55-65.

15. Chobanian AV, Bakris GL, Black HR, et al. Seventh report of the Joint National Committee on Prevention, Detection, Evaluation, and Treatment of High Blood Pressure. Hypertension. 2003;42(6): 1206-52. doi:10.1001/jama.289.19.2560.

16. Stefanick ML, Mackey S, Sheehan M, Ellsworth N, Haskell WL, Wood PD. Effects of diet and exercise in men and postmenopausal women with low levels of HDL cholesterol and high levels of LDL cholesterol. N Engl J Med. 1998;252:12-20. doi:10.1056/ NEJM199807023390103.

17. Semlistsch T, Jeitler K, Hemken LG, et al. Increasing physical activity for the treatment of hypertension: a systematic review and meta-analysis. Sports Med. 2013;43(10):1009-23. doi:10.1007/ s40279-013-0065-6.

18. Matyas E, Jeitler K, Horvath K, et al. Benefit assessment of salt reduction in patients with hypertension: systematic overview. $\mathrm{J}$ Hypertens. 2011;29(5):821-8. doi:10.1097/HJH. 0b013e3283442840.

19. Horvath K, Jeitler K, Siering U, et al. Long-term effects of weightreducing interventions in hypertensive patients: systematic review and meta-analysis. Arch Intern Med. 2008;168(6):571-80. doi:10. 1001/archinte.168.6.571.

20. Appel LJ, Moore TJ, Obarzanek E, et al. Aclinical trial of the effects of dietary patterns on blood pressure. The DASH Collaborative Research Group. N Engl J Med. 1997;336:1117-24. doi:10.1056/ NEJM199704173361601.

21. Whelton PK, He J, Appel LJ, et al. Primary prevention of hypertension: clinical and public health advisory from The National High Blood Pressure Education Program. JAMA. 2002;288(15):1882-8. doi:10.1001/jama.288.15.1882.

22. Thompson PD, Buchner D, Pina IL, et al. Exercise and physical activity in the prevention and treatment of atherosclerotic cardiovascular disease: a statement from the council on clinical cardiology (subcommittee on exercise, rehabilitation, and prevention) and the council on nutrition, physical activity, and metabolism (subcommittee on physical activity). Circulation. 2003;107:3109-16. doi:10. 1161/01.CIR.0000075572.40158.77.

23. Kaholokula JK, Nacapoy AH, Dang KL. Social justice as a public health imperative for Kānaka Maoli. AlterNative: An International Journal of Indigenous Peoples. 2009;5(2):117-37.

24. Kaholokula JK, Townsend CT, Sinclair K, et al. Chapter 24: The PILI 'Ohana Project. In: Brennan VM, Kumanyiki S, Zambrana RE, editors. obesity interventions in underserved U.S. communities: evidence and directions. Baltimore, MD: John Hopkins University Press; 2014.

25. Mau MK, Kaholokula JK, West MR, et al. Translating diabetes prevention into native Hawaiian and Pacific Islander communities: the PILI 'Ohana pilot project. Prog Community Health Partnersh. 2010;4(1):7-16. doi:10.1353/cpr.0.0111.

26. Wang J, Feng B, Yang X, et al. Tai Chi for essential hypertension. Evid Based Conplement Alternat Med. 2013;2013:215254. doi:10. 1155/2013/215254.

27. Kaeppler AL. Hula Pahu: Hawaiian drum dances. Honolulu HI: Bishop Museum Press; 1993.

28. Stillman AK. Sacred Hula: The Historical Hula 'Āla'apapa. Bulletin in Anthropology 8. Honolulu HI: Bishop Museum Press; 1998.

29. Usagawa T, Look M, de Silva M, et al. Metabolic determination in the cultural dance of hula. Int J Sports Med. 2014;35(5):399-402. doi:10.1055/s-0033-1353213.

30. Haskell WL, Lee IM, Pate RR, et al. Physical activity and public health. Updated recommendations for adults from the American College of Sports Medicine and the American Heart Association. Circulation. 2007;116:1081-93. doi:10.1249/mss. 0b013e3180616b27.
31. Lan C, Chen SY, Lai JS, Wong MK. The effect of Tai Chi on cardiopulmonary function in patients with coronary artery bypass surgery. Med Sci Sports Exerc. 1999;31(5):634-8.

32. Yeh GY, Wood MJ, Lorell BH, et al. Effects of Tai Chi mind-body movement therapy on functional status and exercise capacity in patients with chronic heart failure: a randomized controlled trial. Am J Med. 2004;117:541-8. doi:10.1016/j.amjmed.2004.04.016.

33. Morisky DE, DeMuth NM, Field-Fass M, Green LW, Levine DM. Evaluation of family health education to build social support for long-term control of high blood pressure. Health Educ Q. 1985;12(1):35-50.

34. Bell CN, Thorpe Jr RJ, Laveist TA. Race/ethnicity and hypertension: the role of social support. Am J Hypertens. 2010;23(5):534 40. doi:10.1038/ajh.2010.28.

35. Zanini CR, Jardim PC, Salgado CM, et al. Music therapy effects on the quality of life and the blood pressure of hypertensive patients. Arq Bras Cardiol. 2009;93(5):495-500.

36. Pickering TG, Hall JE, Appel LJ, et al. Recommendations for blood pressure measurement in humans and experimental animals: part 1: blood pressure measurement in humans: a statement for professionals from the subcommittee of professional and public education of the American Heart Association council on high blood pressure research. Circulation. 2005;111:697-716. doi:10.1161/01.CIR. 0000154900.76284.F6.

37. Glynn LG, Murphy AW, Smith SM, Schroeder K, Fahey T. Interventions used to improve control of blood pressure in patients with hypertension. Cochrane Database System Review. 2010;3: CD005182. doi:10.1002/14651858.CD005182.pub4.

38. American Thoracic Society. ATS statement: guidelines for the sixminute walk test. Am J Respir Crit Care Med. 2002;166:111-7. doi: 10.1164/ajrccm.166.1.at1102.

39. Kaholokula JK, Townsend CKM, Ige A, et al. Socio-demographic, behavioral, and biological variables related to weight loss in Native Hawaiians and other Pacific Islanders. Obesity. 2013;21:E196-203. doi:10.1002/oby.20038.

40. Ware J, Jr Kosinski M, Keller SD. A 12-Item Short-Form Health Survey: construction of scales and preliminary tests of reliability and validity. Med Care. 1996;34(3):220-33.

41. Busija L, Pausenberger E, Haines TP, Haymes S, Buchbinder R, Osborne RH. Adult measures of general health and health-related quality of life: Medical Outcomes Study Short Form 36-Item (SF36) and Short Form 12-Item (SF-12) Health Surveys, Nottingham Health Profile (NHP), Sickness Impact Profile (SIP), Medical Outcomes Study Short Form 6D (SF-6D), Health Utilities Index Mark 3 (HUI3), Quality of Well-Being Scale (QWB), and Assessment of Quality of Life (AQoL). Arthritis Care Res . 2011;63(S11):S383-412. doi:10.1002/acr.20541.

42. Heart Failure Society of America. Heart failure education modules. Reprinted 2006. http://www.hfsa.org/heart_failure_education modules.asp. Accessed March 28, 2014.

43. Thompson PD, Arena R, Riebe D, Pescatello LS. American College of Sports Medicine. ACSM's new preparticipation health screening recommendations from ACSM's guidelines for exercise testing and prescription, ninth edition. Curr Sports Med Rep. 2013;12(4):215-7. doi:10.1249/JSR.0b013e31829a68cf.

44. Pashkow FJ, Dafoe WA. Clinical cardiac rehabilitation, a cardiologist's guide. 2nd ed. Baltimore, MD: Williams \& Wilkins; 1999.

45. Bandura A. Social foundations of thought and action: a socialcognitive theory. Englewood Cliffs, NJ: Prentice-Hall; 1986.

46. Young MD, Plotnikoff RC, Collins CE, Callister R, Morgan PJ. Social cognitive theory and physical activity: a systematic review and meta-analysis. Obes Rev. 2014;15(12):983-95. doi:10.1111/ obr. 12225 .

47. Wang J, Feng B, Yang X, Liu W, Teng F, Li S, et al. Tai Chi for essential hypertension. Evid Based Complement Alternat Med. 2013;2013:215254. doi:10.1155/2013/215254. 
48. Yeh GY, Wang C, Wayne PM, Phillips RS. The effect of tai chi exercise on blood pressure: a systematic review. Prev Cardiol. 2008;11(2):82-9.

49. Harris PA, Taylor R, Thielke R, Payne J, Gonzalez N, Conde JG. Research electronic data capture (REDCap) - a metadata-driven methodology and workflow process for providing translational research informatics support. J Biomed Inform. 2009;42(2):377-81. doi:10.1016/j.jbi.2008.08.010.

50. Law M, Wald N, Morris J. Lowering blood pressure to prevent myocardial infarction and stroke. Health Technol Assess. 2003;7(31):1-94. doi:10.3310/hta7310.

51. Neal B, MacMahon S, Chapman N. Effects of ACE inhibitors, calcium antagonists, and other blood-pressure-lowering drugs: results of prospectively designed overviews of randomised trials. Blood Pressure Lowering Treatment Trialists' Collaboration. Lancet. 2000;356(9246):1955-64. doi:10.1016/S0140-6736(00) 03307-9.

52. Sutoo D, Akiyama K. Music improves dopaminergic neurotransmission: demonstration based on the effect of music on blood pressure regulation. Brain Res. 2004;1016(2):255-62. doi:10.1016/j. brainres.2004.05.018.

53. Uchino BN, Cacioppo JT, Kiecolt-Glaser JK. The relationship between social support and physiological processes: a review with emphasis on underlying mechanisms and implications for health. Psychol Bull. 1996;119(3):48-531. doi:10.1037/0033-2909.119.3. 488. 